\title{
Technical note: Evaluation of the diagnostic accuracy of 2 point-of-care $\beta$-hydroxybutyrate devices in stored bovine plasma at room temperature and at $37^{\circ} \mathrm{C}$
}

\author{
F. A. Leal Yepes, ${ }^{*}$ D. V. Nydam, ${ }^{*}$ W. Heuwieser, ${ }^{*} \dagger$ and S. Mann ${ }^{* 1}$ \\ *Department of Population Medicine and Diagnostic Sciences, College of Veterinary Medicine, Cornell University, Ithaca, NY 14853 \\ †Clinic for Animal Reproduction, Faculty of Veterinary Medicine, Freie Universität Berlin, Koenigsweg 65, 14163 Berlin, Germany
}

\begin{abstract}
The use of point-of-care (POC) devices to measure blood metabolites, such as $\beta$-hydroxybutyrate (BHB), on farm have become an important diagnostic and screening tool in the modern dairy industry. The POC devices allow for immediate decision making and are often more economical than the use of laboratory-based methods; however, precision and accuracy may be lower when measurements are performed in an uncontrolled environment. Ideally, the advantages of the POC devices and the standardized laboratory environment could be combined when measuring samples that do not require an immediate result - for example, in research applications or when immediate intervention is not the goal. The objective of this study was to compare the capability of 2 POC devices (TaiDoc, Pharmadoc, Lübeck, Germany; Precision Xtra, Abbott Diabetes Care, Abingdon, UK) to measure BHB concentrations either at room temperature $\left(\mathrm{RT} ; 20-22^{\circ} \mathrm{C}\right)$ or at $37^{\circ} \mathrm{C}$ compared with the gold standard test in stored plasma samples. Whole blood from multiparous Holstein dairy cows $(\mathrm{n}=113)$ was sampled from the coccygeal vessels between $28 \mathrm{~d}$ before expected calving and 42 DIM. Whole-blood BHB concentrations were determined cow-side using the TaiDoc POC device. Plasma was separated within $1 \mathrm{~h}$ of collection and stored until analysis. A subset of stored plasma samples $(\mathrm{n}=100)$ consisting of 1 sample per animal was chosen retrospectively based on the BHB concentrations in whole blood within the range of 0.2 to $4.0 \mathrm{mmol} / \mathrm{L}$. The samples were analyzed for BHB plasma concentration using an automated chemistry analyzer (Hitachi 917, Hitachi, Tokyo, Japan), which was considered the gold standard. On the same day, the samples were also measured with the 2 POC devices, with samples either at RT or
\end{abstract}

Received October 8, 2017.

Accepted March 15, 2018.

${ }^{1}$ Corresponding author: sm682@cornell.edu heated up to $37^{\circ} \mathrm{C}$. Our study showed high Spearman correlation coefficients $(>0.99)$ using either device and with samples at both temperatures compared with the gold standard. Passing-Bablok regression revealed a very strong correlation $(>0.99)$, indicating good agreement between both POC devices and the gold standard method. For hyperketonemia detection, defined as BHB concentration $\geq 1.2 \mathrm{mmol} / \mathrm{L}$, the sensitivity for both POC devices at RT and $37^{\circ} \mathrm{C}$ was equally high at $100 \%$. Specificity was lowest (67.4\%) for the TaiDoc used with plasma at RT and was highest (86.5\%) when plasma was measured at $37^{\circ} \mathrm{C}$ with the Precision Xtra meter. Bland-Altman plots revealed a mean bias of 0.25 and $0.4 \mathrm{mmol} / \mathrm{L}$ for the Precision Xtra meter and TaiDoc, respectively, when tested on plasma at $37^{\circ} \mathrm{C}$. Our data showed that both POC devices are suitable for measuring BHB concentration in stored bovine plasma, and accuracy was highest when samples were heated to $37^{\circ} \mathrm{C}$ compared with RT.

Key words: $\beta$-hydroxybutyrate, point of care, hyperketonemia, plasma

\section{Technical Note}

Subclinical hyperketonemia (HYK) in dairy cows has been defined as a BHB concentration in blood of $\geq 1.2 \mathrm{mmol} / \mathrm{L}$ (Oetzel, 2004; McArt et al., 2011). The gold standard spectrophotometric test for the determination of BHB concentrations in either plasma or serum is performed under controlled laboratory and environmental conditions. Each test costs $\$ 13$ (as of December 2017) at the New York Animal Health Diagnostic Center (AHDC; Ithaca NY). Consequently, a lower-cost method with accuracy and precision comparable to the gold standard is preferable for routine measurement of BHB plasma concentration in the dairy industry. Several point-of-care (POC) devices were previously validated as cow-side methods to measure BHB concentration in bovine whole blood (Iwersen et al., 2009; Bach et al., 2016). Because of their user-friendliness and low maintenance and calibration requirements, 
POC devices are valuable tools in the field. Moreover, such a method may allow retrospective sample analysis under controlled environmental conditions (e.g., in research applications; Pineda and Cardoso, 2015) or when immediate results are not needed. Often, plasma is the sample of choice for storage, but available POC devices are calibrated and designed for use on whole blood, requiring validation of the use of this sample type in such applications. The Precision Xtra (Abbott Diabetes Care, Abingdon, UK) has been widely used as a diagnostic tool for HYK in the field (McArt et al., 2011; Mann et al., 2015) and was recently validated as a feasible method for estimating BHB concentration in bovine plasma and serum (Pineda and Cardoso, 2015). The approximate cost for each BHB test using the Precision Xtra was $\$ 1.70$ to $\$ 4.00$ (LeBlanc, 2010) plus the cost of the meter. Currently, the Precision Xtra test strips are available and sold only for human use in the United States, thus increasing the cost and reducing access for veterinary applications.

Recently, the TaiDoc POC device (Pharmadoc, Lübeck, Germany) was shown to measure BHB concentration in bovine whole blood with good accuracy and precision when testing was performed at room temperature (RT; Bach et al., 2016). The current cost for each TaiDoc strip is approximately $\$ 1.10$, which makes this a convenient and cost-effective method for BHB testing. However, the temperature range for the TaiDoc meter is 5 to $40^{\circ} \mathrm{C}$, similar to the Precision Xtra $\left(10-50^{\circ} \mathrm{C}\right)$. Using POC devices on farm under conditions that differ from manufacturer specifications may lead to unknown variation in accuracy and precision due to limitations in temperature, humidity, and sample quality. A possible solution is to combine the ease and cost-effectiveness of POC devices with analysis in a controlled environment after sample collection. Plasma samples are easily obtained and BHB concentrations are stable in this sample type (Stokol and Nydam, 2005).

The objective of this study was to compare the results of the TaiDoc POC device for BHB concentrations in plasma either at $\mathrm{RT}$ or at $37^{\circ} \mathrm{C}$ with the results obtained with the gold standard method and a second POC device previously described for use in plasma samples (Precision Xtra; Pineda and Cardoso, 2015). Room temperature and $37^{\circ} \mathrm{C}$ were selected because laboratory tests are often carried out at either of the 2 temperatures. The $37^{\circ} \mathrm{C}$ temperature was selected to closely reproduce the conditions when using fresh blood samples on POC devices as well as to approximate the gold standard methodology.

All procedures for this experiment were approved by the Cornell University Institutional Animal Care and Use Committee (protocol no. 2014-0118 and
2015-0097). Holstein dairy cows $(\mathrm{n}=113)$ from the Cornell University Ruminant Center (Harford, NY) were enrolled between January 2016 and July 2016. All cows were entering second or greater lactation and were sampled from $28 \mathrm{~d}$ before expected calving date until 42 DIM. Whole blood was collected 3 times per week from the coccygeal vessels using 10-mL blood collection tubes (Becton Dickinson, Franklin Lakes, NJ) containing 158 USP of sodium heparin and 20-gauge $\times 2.54-\mathrm{cm}$ blood collection needles. Concentration of BHB was measured immediately after sample collection in whole blood using the TaiDoc POC device (Bach et al., 2016). After measurements were completed, blood samples were placed on ice and plasma was separated within $1 \mathrm{~h}$ of sample collection at 3,000 $\times \mathrm{g}$ for $20 \mathrm{~min}$ at $4^{\circ} \mathrm{C}$ and stored in aliquots at $-20^{\circ} \mathrm{C}$ until analysis.

A subset of plasma samples $(\mathrm{n}=100)$ was chosen retrospectively based on the $\mathrm{BHB}$ concentrations in whole blood obtained with the TaiDoc POC device. Samples were included in this study based on whole blood BHB concentrations in increments of $0.1 \mathrm{mmol} / \mathrm{L}$ ranging from 0.2 to $4.0 \mathrm{mmol} / \mathrm{L}$, with no more than 4 samples per increment. The plasma sample subset did not include more than 1 sample per animal to ensure independence of observations for statistical analysis. Samples were allowed to thaw on ice and were submitted to the AHDC for measurement of BHB concentration using a commercially available test kit (D-3 Hydroxybutyrate Ranbut, Randox Laboratories, Antrim, UK) on an automated chemistry analyzer at $37 \pm 0.2^{\circ} \mathrm{C}$ (Hitachi 917, Hitachi, Tokyo, Japan) as the gold standard. The BHB plasma concentrations were measured on the same day as the gold standard and on the same aliquot with both POC devices as submitted to the AHDC. Meters were used at all times at RT $\left(20-22^{\circ} \mathrm{C}\right)$. Plasma samples were measured first at RT with both devices and then at $37^{\circ} \mathrm{C}$ after heating plasma samples for at least $5 \mathrm{~min}$ in a water bath.

Data analysis was performed in SAS 9.4 (SAS Institute Inc., Cary, NC). Correlation coefficients (Spearman) between BHB gold standard and BHB concentrations obtained with both POC devices and samples at either RT or $37^{\circ} \mathrm{C}$ were computed using PROC CORR (SAS 9.4). Coefficient of variation $(\mathbf{C V} ; \%)$ was determined for 1 sample each in the low $(0.8 \mathrm{mmol} / \mathrm{L})$ and high $(3.9 \mathrm{mmol} / \mathrm{L})$ range of our data based on the gold standard method. For each sample, 12 measurements were performed with each POC device and with the sample at either $\mathrm{RT}$ or $37^{\circ} \mathrm{C}$. Sensitivity of each method was calculated as the proportion of animals properly diagnosed as positive for HKY (BHB plasma concentration $\geq 1.2 \mathrm{mmol} / \mathrm{L}$ ) among all animals identified as positive by each POC device compared with those clas- 
sified as positive by the gold standard. Specificity was calculated as the proportion of animals properly diagnosed as negative for HKY among all animals identified as negative by each POC device compared with those classified as negative by the gold standard. The $95 \%$ CI was calculated for sensitivity and specificity values using PROC FREQ in SAS. Regression analysis between both methods with samples at RT and $37^{\circ} \mathrm{C}$ and the gold standard was performed using PROC REG in SAS. Linearity between methods was visually assessed using the plot of residuals. Passing-Bablok regression coefficients, slopes, and intercepts were obtained using MedCalc Statistical Software version 17.8.6 (MedCalc Software, Ostend, Belgium). This regression fits a straight line to 2 variables and is less sensitive to outliers because it assumes measurement errors in both methods. Perfect agreement yields an intercept of 0 and a slope of 1 . Bland-Altman plots were generated with GraphPad Prism (v. 7.02, GraphPad Software, La Jolla, CA) to graphically demonstrate the level of agreement between 2 tests that cannot be captured by the correlation estimate alone (Bland and Altman, 1986). The Bland-Altman plot includes a solid horizontal line showing the mean bias between the 2 methods as well as the $95 \%$ confidence interval of agreement.

Out of the 100 plasma samples used in this experiment, 57 had a BHB plasma concentration $\geq 1.2$ $\mathrm{mmol} / \mathrm{L}$ as assessed by the gold standard method. Data of BHB plasma concentrations from the gold standard test showed a nonparametric distribution. The median was $1.3 \mathrm{mmol} / \mathrm{L}$, ranging from 0.1 to $5.5 \mathrm{mmol} / \mathrm{L}$. Spearman correlation coefficients of the measured BHB plasma concentrations between the gold standard and both POC devices at both plasma temperatures were $>0.99$. Similar coefficients of correlation were reported for the Precision Xtra (Pearson $r=0.99$ ) on bovine plasma (Pineda and Cardoso, 2015) and for the Optium Xceed POC meter (Abbott Diabetes Care) in $\mathrm{K}_{3}$-EDTA anticoagulated bovine whole blood compared with analysis of plasma using the laboratory gold standard method (Pearson $\mathrm{r}=0.97$; Voyvoda and Erdogan, 2010). Additionally, the correlation coefficients in our study for both POC devices in plasma either at RT or at $37^{\circ} \mathrm{C}$ were comparable with other studies using whole blood - for example, 0.95 (Iwersen et al., 2009) and 0.97 (Bach et al., 2016). The CV were generally lower when samples were heated to $37^{\circ} \mathrm{C}$. The $\mathrm{CV}$ for the sample at $0.8 \mathrm{mmol} / \mathrm{L}$ were $11,4,12$, and $9 \%$ for TaiDoc with plasma at RT, TaiDoc with plasma at $37^{\circ} \mathrm{C}$, Precision Xtra with plasma at RT, and Precision Xtra with plasma at $37^{\circ} \mathrm{C}$, respectively. The $\mathrm{CV}$ for the sample at $3.9 \mathrm{mmol} / \mathrm{L}$ were $2,2,7$, and $5 \%$ for the 2 devices and temperatures, respectively.
Sensitivity and specificity of both POC devices for the classification of HYK are summarized in Table 1. The TaiDoc and Precision Xtra measurements of plasma at both RT and $37^{\circ} \mathrm{C}$ had perfect sensitivity for HYK. The greatest specificity for HYK was shown for Precision $\mathrm{Xtra}$ and TaiDoc at $37^{\circ} \mathrm{C}$ sample temperature. A higher sensitivity for Precision Xtra and TaiDoc meters was demonstrated in this study compared with a sensitivity of 0.85 described by Voyvoda and Erdogan (2010) but similar to other studies using bovine whole blood, plasma, and serum (Iwersen et al., 2009; Pineda and Cardoso, 2015; Bach et al., 2016). Moreover, specificity values presented here were higher than the specificity of 0.51 previously reported by Pineda and Cardoso (2015) but were lower than the values found in several other studies (Iwersen et al., 2009; Voyvoda and Erdogan, 2010). The temperature of whole blood, serum, and plasma can affect the results for the concentration of metabolites such as nonesterified fatty acids (Stokol and Nydam, 2005), glucose (Megahed et al., 2015), and BHB (Iwersen et al., 2013; Megahed et al., 2015). This may also be the reason for the superior CV results for samples heated to $37^{\circ} \mathrm{C}$ in this study. Differences in sensitivity and specificity may be attributed to different temperatures in our and other studies. It has been shown that when POC devices and strips are exposed to temperatures out of the range specified by the manufacturer, erroneous measurements might result (Nerhus et al., 2011; Deakin et al., 2015).

Linear regression is traditionally performed when comparing 2 methods with continuous outcomes, but this technique assumes normality of data distribution, errors, and absence of error in the gold standard. Linear regression analysis revealed a linear relationship between both methods with plasma at $\mathrm{RT}$ and $37^{\circ} \mathrm{C}$ and the gold standard (Figure 1). The TaiDoc meter used with plasma at $37^{\circ} \mathrm{C}$ had the greatest linear relationship with the gold standard test $\left(\mathrm{R}^{2}=0.97\right)$. However, when linearity and normality were graphically assessed using the plot of residuals, a deviation from both linearity and normality was observed; this suggested lack of fit for all linear regression models, making this a less desirable technique for method comparison in this case. This can be caused by random error within both the gold standard method and POC devices or intermittent imprecision from the POC devices (Stöckl et al., 1998). Therefore, we explored Passing-Bablok regression because this allows us to compare methods with nonparametric distributed data (Table 2); however, this method also assumes linearity between the 2 variables. The intercept is interpreted as the systematic mean bias between the 2 methods, whereas the slope indicates proportional bias between 2 methods. 
Table 1. Performance of 2 point-of-care test devices for the classification of hyperketonemia, defined as BHB plasma concentration $\geq 1.2 \mathrm{mmol} / \mathrm{L}$ at room temperature or $37^{\circ} \mathrm{C}(\mathrm{n}=100)$

\begin{tabular}{llcc}
\hline Test $^{1}$ & $\begin{array}{l}\text { Plasma } \\
\text { temperature }\end{array}$ & $\begin{array}{c}\text { Sensitivity }^{2} \\
(95 \% \mathrm{CI})\end{array}$ & $\begin{array}{c}\text { Specificity }^{4} \\
(95 \% \mathrm{CI})\end{array}$ \\
\hline TaiDoc & $\mathrm{RT}$ & $100.0(93.7,100.0)$ & $67.4(51.4,80.9)$ \\
TaiDoc & $37^{\circ} \mathrm{C}$ & $100.0(93.7,100.0)$ & $76.4(61.3,88.2)$ \\
Precision Xtra & $\mathrm{RT}$ & $100.0(93.7,100.0)$ & $74.4(58.8,86.4)$ \\
Precision Xtra & $37^{\circ} \mathrm{C}$ & $100.0(93.7,100.0)$ & $86.5(72.0,94.7)$ \\
\hline
\end{tabular}

${ }^{1}$ TaiDoc manufactured by Pharmadoc, Lübeck, Germany. Precision Xtra manufactured by Abbott Diabetes Care, Abingdon, UK.

${ }^{2} \mathrm{BHB}$ concentration was measured in the same aliquot of plasma at room temperature $\left(\mathrm{RT} ; 20-22^{\circ} \mathrm{C}\right)$ and at $37^{\circ} \mathrm{C}$.

${ }^{3}$ Sensitivity (\%) was calculated as the proportion of animals that were classified as positive for hyperketonemia by the meter compared with the animals classified as positive with the gold standard method (BHB plasma concentration $\geq 1.2 \mathrm{mmol} / \mathrm{L}$ ) performed at the New York Animal Health Diagnostic Center (Ithaca, NY).

${ }^{4}$ Specificity (\%) was calculated as the proportion of animals that were classified as negative for hyperketonemia by the meter compared with the number of animals classified as negative by the gold standard method (BHB plasma concentration $<1.2 \mathrm{mmol} / \mathrm{L}$ ) performed at the New York Animal Health Diagnostic Center (Ithaca, NY).

A

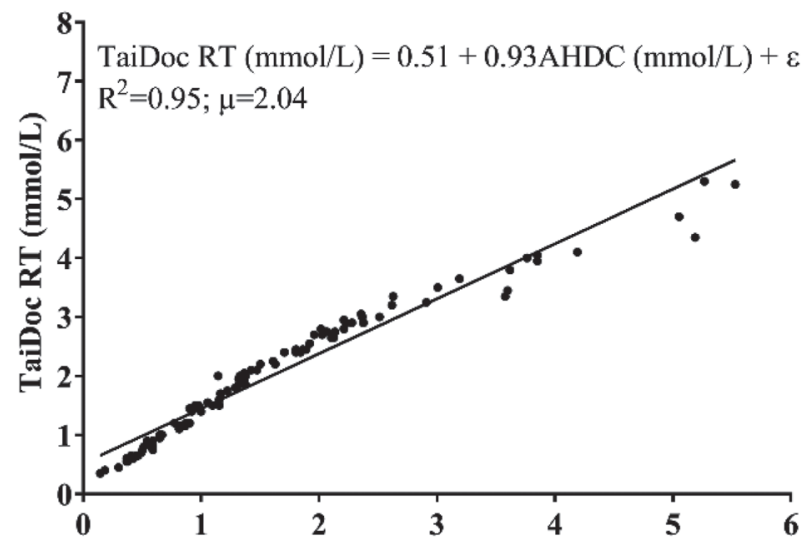

C

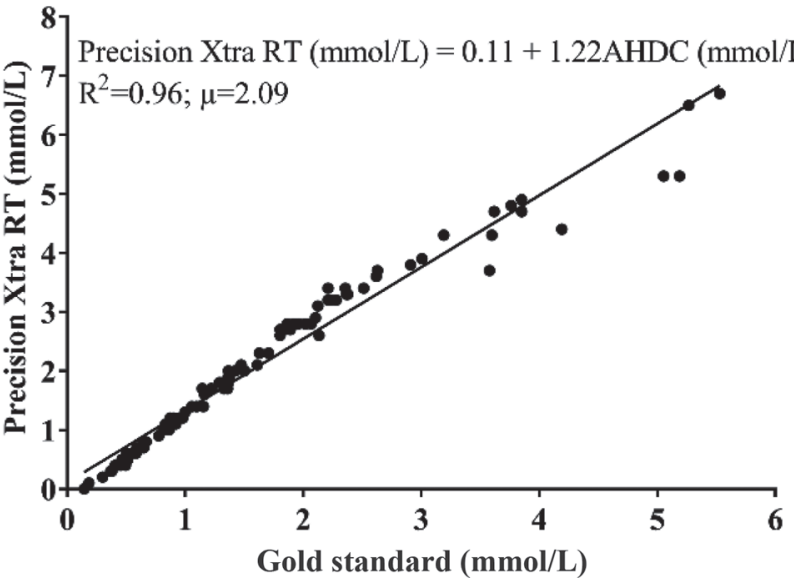

B

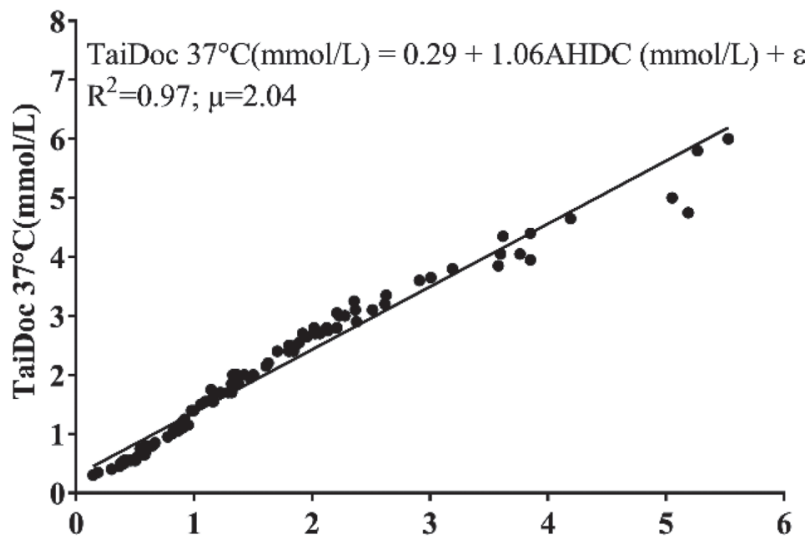

D

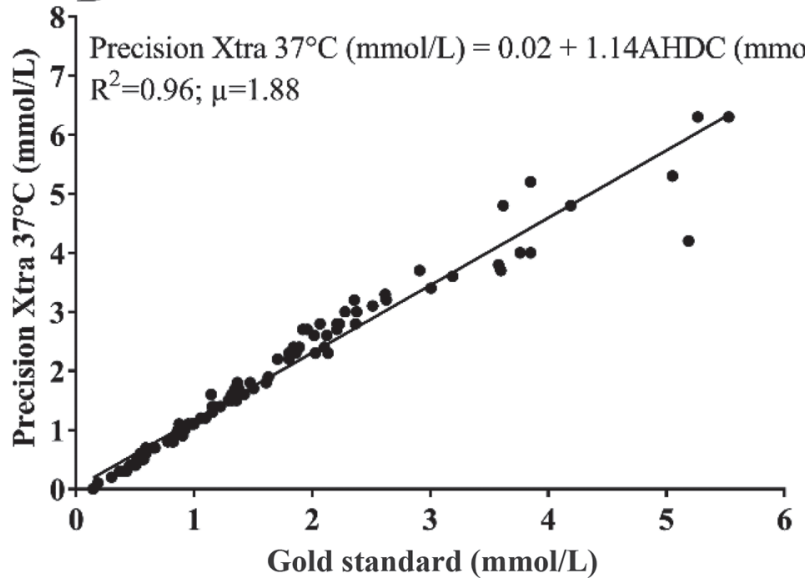

Figure 1. Linear regression analysis between BHB concentrations measured in plasma with the gold standard method (Randox Laboratories, Antrim, UK) and (A) TaiDoc (Pharmadoc, Lübeck, Germany) in plasma at room temperature (RT; $20-22^{\circ} \mathrm{C}$ ), (B) TaiDoc in plasma at $37^{\circ} \mathrm{C}$, (C) Precision Xtra (Abbott Diabetes Care, Abingdon, UK) in plasma at RT, and (D) Precision Xtra in plasma at $37^{\circ} \mathrm{C}$. The values obtained by New York Animal Health Diagnostic Center (AHDC) were used as the gold standard test. $\mu=$ the mean of all BHB concentrations measured in plasma with each device. 
Table 2. Passing-Bablok regression coefficients for BHB concentrations in 100 plasma samples at room temperature or $37^{\circ} \mathrm{C}$ measured with 2 point-of-care test devices compared with the gold standard test performed at the New York Animal Health Diagnostic Center (Ithaca, NY)

\begin{tabular}{|c|c|c|c|}
\hline Test $^{1}$ & $\begin{array}{l}\text { Plasma } \\
\text { temperature }^{2}\end{array}$ & $\begin{array}{l}\text { Intercept } \\
(95 \% \text { CI })\end{array}$ & $\begin{array}{c}\text { Slope } \\
(95 \% \text { CI })\end{array}$ \\
\hline TaiDoc & $\mathrm{RT}$ & $0.22(0.16,0.30)$ & $1.17(1.10,1.20)$ \\
\hline TaiDoc & $37^{\circ} \mathrm{C}$ & $0.05(-0.01,0.11)$ & $1.26(1.20,1.32)$ \\
\hline Precision Xtra & $\mathrm{RT}$ & $-0.19(-0.23,-0.14)$ & $1.46(1.41,1.50)$ \\
\hline Precision Xtra & $37^{\circ} \mathrm{C}$ & $-0.18(-0.21,-0.14)$ & $1.31(1.26,1.34)$ \\
\hline
\end{tabular}

${ }^{1}$ TaiDoc manufactured by Pharmadoc, Lübeck, Germany. Precision Xtra manufactured by Abbott Diabetes Care, Abingdon, UK.

${ }^{2} \mathrm{BHB}$ concentration was measured in the same aliquot of plasma at room temperature $\left(\mathrm{RT} ; 20-22^{\circ} \mathrm{C}\right)$ and at $37^{\circ} \mathrm{C}$
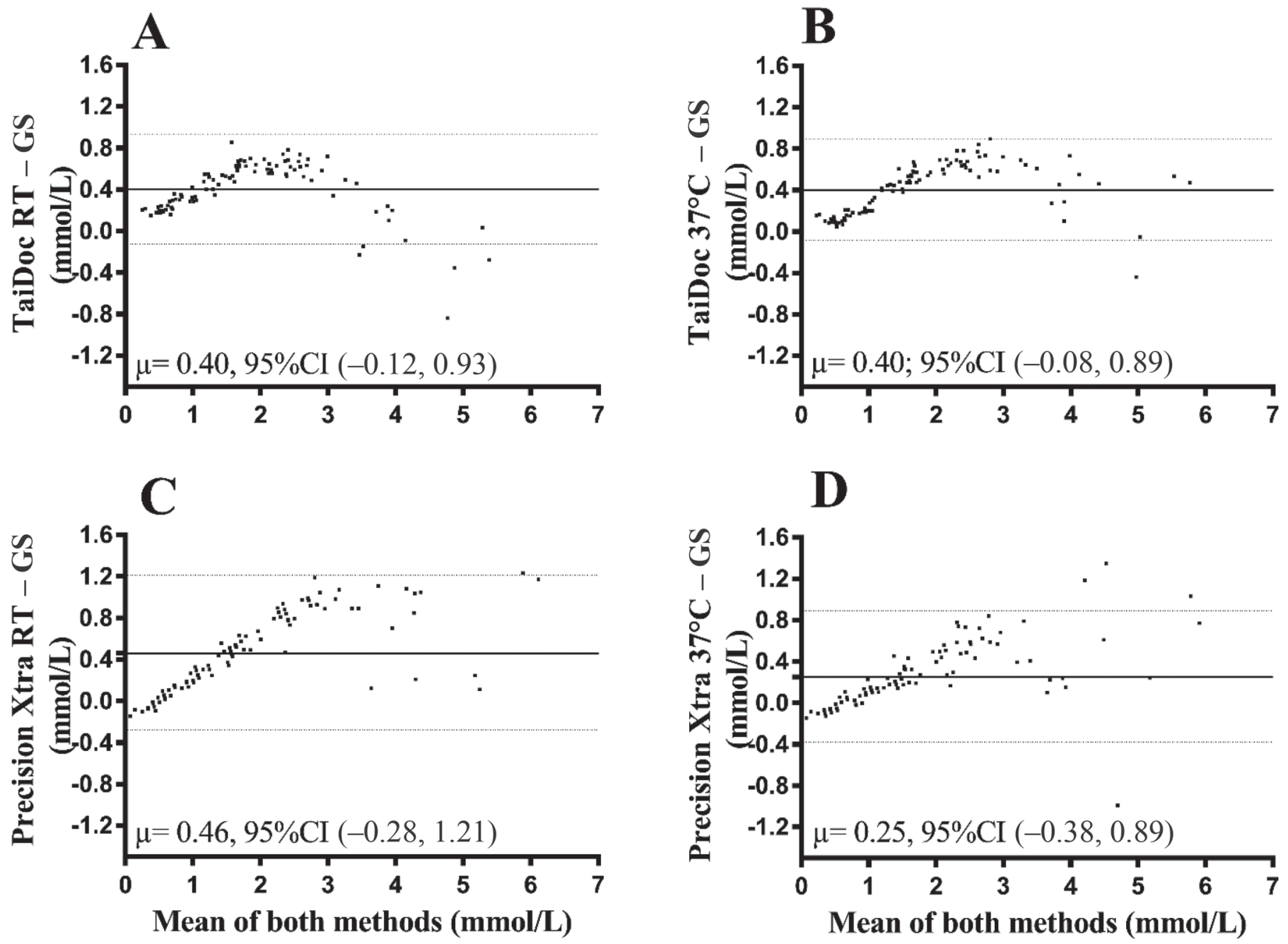

Figure 2. Bland-Altman plots of the difference in BHB concentrations in plasma between 2 tests against their mean and (A) TaiDoc (Pharmadoc, Lübeck, Germany) at room temperature (RT; $20-22^{\circ} \mathrm{C}$ ) versus the gold standard (GS; Randox Laboratories, Antrim, UK), (B) TaiDoc at $37^{\circ} \mathrm{C}$ versus the GS, (C) Precision Xtra (Abbott Diabetes Care, Abingdon, UK) at room temperature (RT) versus the GS, and (D) Precision Xtra at $37^{\circ} \mathrm{C}$ versus the GS. The solid horizontal line represents the mean bias; horizontal dashed lines represent the $95 \%$ CI of agreement. $\mu=$ the overall mean bias (mmol/L) calculated as point-of-care device (either TaiDoc or PrecisionXtra) minus the GS. A positive mean bias shows an overestimation of the BHB concentration by the point-of-care device compared with the GS. 
The Spearman's correlation coefficients obtained from the Passing-Bablok regressions showed a strong correlation $(>0.99)$ between the gold standard and the 2 POC devices. According to Passing and Bablok (1983), there is no constant difference between the 2 methods if the $95 \%$ confidence interval of the intercept includes zero. Therefore, among all comparisons the TaiDoc in plasma at $37^{\circ} \mathrm{C}$ (intercept $0.05 ; 95 \%$ CI: $-0.01,0.11$ ) was the only POC device without a constant difference compared with the gold standard.

The test agreement between BHB plasma concentrations measured by the gold standard and both POC devices is illustrated in Figure 2. The Precision Xtra and the TaiDoc devices with samples at $37^{\circ} \mathrm{C}$ had a mean bias (i.e., the mean difference in accuracy of the tested methods compared with the gold standard) of $0.25 \mathrm{mmol} / \mathrm{L}(95 \% \mathrm{CI}:-0.38,0.89)$ and $0.40 \mathrm{mmol} / \mathrm{L}$ (95\% CI: - 0.99, 0.99), respectively, when compared with the gold standard. Although the TaiDoc had a larger bias, the $95 \%$ confidence limits of agreement for TaiDoc on plasma at $37^{\circ} \mathrm{C}$ were smaller than those of the Precision Xtra. The mean bias that we determined for both POC devices in this study was in agreement with prior reports using Precision Xtra in bovine plasma (Voyvoda and Erdogan, 2010; Pineda and Cardoso, 2015; Bach et al., 2016). Users of POC devices should be aware of the bias in BHB concentrations compared with the gold standard, particularly when BHB concentrations increase. For both meters and either sample temperature the bias was positive, meaning that $\mathrm{BHB}$ concentrations were generally overestimated by these methods compared with the gold standard. The mean bias for the TaiDoc and Precision Xtra devices was similar to the values reported recently for whole blood samples $(0.34$ and $0.21 \mathrm{mmol} / \mathrm{L}$, respectively). However, given that the method comparisons showed deviation from linearity, samples in the low-to-mid range of BHB concentration were overestimated for the TaiDoc, whereas samples with the highest BHB concentrations were underestimated by this device, particularly at RT. In contrast, measurements of BHB in plasma with the Precision Xtra seemed to overestimate concentrations for most sample concentrations, with the bias increasing as concentrations measured with the gold standard increased.

Based on the sensitivity and specificity, the tested POC devices can be used with satisfactory accuracy to classify samples as hyperketonemic or nonhyperketonemic in bovine plasma when measured at $37^{\circ} \mathrm{C}$. The precision of both devices was higher with samples at $37^{\circ} \mathrm{C}$ compared with RT based on the $\mathrm{CV}$. Mean bias was comparable with results previously reported in whole blood for both meters at both temperatures.
When POC devices are used, sample temperature plays an important role. In conclusion, this work provides evidence that POC devices can be used with stored plasma samples. This enables the use of POC devices in research applications when such samples are available for BHB measurements instead of fresh whole blood.

\section{ACKNOWLEDGMENTS}

The authors thank Pharmadoc (Lübeck, Germany) and TaiDoc (New Taipei City, Taiwan) for providing the POC devices and test strips. This study was supported by the Agriculture and Food Research Initiative competitive grant no. 2012-67015-30230 from the USDA National Institute of Food and Agriculture (Washington, DC).

\section{REFERENCES}

Bach, K. D., W. Heuwieser, and J. A. McArt. 2016. Technical note: Comparison of 4 electronic handheld meters for diagnosing hyperketonemia in dairy cows. J. Dairy Sci. 99:9136-9142. https://doi .org/10.3168/jds.2016-11077.

Bland, J. M., and D. Altman. 1986. Statistical methods for assessing agreement between two methods of clinical measurement. Lancet 1:307-310. https://doi.org/10.1016/S0140-6736(86)90837-8.

Deakin, S., D. Steele, S. Clarke, C. Gribben, A.-M. Bexley, R. Laan, and D. Kerr. 2015. Cook and chill. J. Diabetes Sci. Technol. 9:1260-1269. https://doi.org/10.1177/1932296815598775.

Iwersen, M., U. Falkenberg, R. Voigtsberger, D. Forderung, and W. Heuwieser. 2009. Evaluation of an electronic cowside test to detect subclinical ketosis in dairy cows. J. Dairy Sci. 92:2618-2624. https://doi.org/10.3168/jds.2008-1795.

Iwersen, M., D. Klein-Jöbstl, M. Pichler, L. Roland, B. Fidlschuster, I. Schwendenwein, and M. Drillich. 2013. Comparison of 2 electronic cowside tests to detect subclinical ketosis in dairy cows and the influence of the temperature and type of blood sample on the test results. J. Dairy Sci. 96:7719-7730. https://doi.org/10.3168/ jds.2013-7121.

LeBlanc, S. 2010. Monitoring metabolic health of dairy cattle in the transition period. J. Reprod. Dev. 56:S29-S35. https://doi.org/10 $.1262 /$ jrd.1056S29.

Mann, S., F. A. L. Yepes, T. R. Overton, J. J. Wakshlag, A. L. Lock, C. M. Ryan, and D. V. Nydam. 2015. Dry period plane of energy: Effects on feed intake, energy balance, milk production, and composition in transition dairy cows. J. Dairy Sci. 98:3366-3382. https://doi.org/10.3168/jds.2014-9024.

McArt, J. A. A., D. V. Nydam, P. A. Ospina, and G. R. Oetzel. 2011. A field trial on the effect of propylene glycol on milk yield and resolution of ketosis in fresh cows diagnosed with subclinical ketosis. J. Dairy Sci. 94:6011-6020. https://doi.org/10.3168/jds.2011 $-4463$.

Megahed, A. A., M. W. Hiew, J. R. Townsend, J. B. Messick, and P. D. Constable. 2015. Evaluation of an electrochemical point-of-care meter for measuring glucose concentration in blood from periparturient dairy cattle. J. Vet. Intern. Med. 29:1718-1727. https://doi .org/10.1111/jvim.13608.

Nerhus, K., P. Rustad, and S. Sandberg. 2011. Effect of ambient temperature on analytical performance of self-monitoring blood glucose systems. Diabetes Technol. Ther. 13:883-892. https://doi .org/10.1089/dia.2010.0255.

Oetzel, G. R. 2004. Monitoring and testing dairy herds for metabolic disease. Vet. Clin. North Am. Food Anim. Pract. 20:651-674. https://doi.org/10.1016/j.cvfa.2004.06.006. 
Passing, H., and W. Bablok. 1983. A new biometrical procedure for testing the equality of measurements from two different analytical methods. Application of linear regression procedures for method comparison studies in clinical chemistry, Part I. J. Clin. Chem. Clin. Biochem. 21:709-720. https://doi.org/10.1515/cclm.1983.21 .11.709.

Pineda, A., and F. C. Cardoso. 2015. Technical note: Validation of a handheld meter for measuring $\beta$-hydroxybutyrate concentrations in plasma and serum from dairy cows. J. Dairy Sci. 98:8818-8824. https://doi.org/10.3168/jds.2015-9667.

Stöckl, D., K. Dewitte, and L. M. Thienpont. 1998. Validity of linear regression in method comparison studies: Is it limited by the sta- tistical model or the quality of the analytical input data? Clin. Chem. 44:2340-2346.

Stokol, T., and D. V. Nydam. 2005. Effect of anticoagulant and storage conditions on bovine nonesterified fatty acid and $\beta$-hydroxybutyrate concentrations in blood. J. Dairy Sci. 88:3139-3144. https://doi .org/10.3168/jds.S0022-0302(05)72996-9.

Voyvoda, H., and H. Erdogan. 2010. Use of a hand-held meter for detecting subclinical ketosis in dairy cows. Res. Vet. Sci. 89:344-351. https://doi.org/10.1016/j.rvsc.2010.04.007. 